\title{
LEARNING TO REOPTIMIZE CONSUMPTION AT NEW INCOME LEVELS: A RATIONALE FOR PROSPECT THEORY
}

\author{
Markus K. Brunnermeier \\ Princeton University
}

\begin{abstract}
This paper provides a theoretical rationale for three experimental results of Prospect Theory: risk preferences are over gains and losses, loss aversion, and diminishing sensitivity. We consider a (boundedly rational) decision maker who does not find her new optimal consumption bundle with certainty when she is faced with a new income level. This alters her indirect utility function and makes her more risk averse at her current reference income level and less risk averse for a range of incomes below her reference income level. (JEL: D11)
\end{abstract}

\section{Introduction}

This paper provides a simple and novel explanation for the following widely accepted experimental findings on attitudes towards risk:

(i) Risk preferences depend highly on the individual's reference income level. The reference income plays an important role in determining whether a lottery will be accepted or not. Therefore, it is useful to consider losses and gains with respect to this reference income level rather than to the absolute income level.

(ii) Individuals' preferences exhibit loss aversion, that is, they are much more responsive to losses than to gains. In terms of a von Neumann-Morgenstern utility function, this means that a decrease in income by $\$ x$ results in a much higher utility loss than the utility gain associated with an increase in income by $\$ x$. In other words, the value function is steeper in the loss region than in the gain region. This leads to high risk aversion at the reference income level.

Acknowledgments: An earlier version of this paper was circulated under the title "On Bounded Rationality and Risk Aversion." I benefited from suggestions from Margaret Bray, Kevin Roberts and three anonymous referees. I am also grateful for comments from Smita B. Brunnermeier, Bart Lipman, Volker Nocke, Rohini Pande, Ben Polak, Sven Rady, Sönje Reiche, Ariel Rubinstein, Klaus M. Schmidt, Patrick Schmitz, Mimi Tan, David E. Wildasin, and two anonymous referees. Any errors are my own. Financial support from the University of Bonn, European Union, CEPS summer support at Princeton University and NSF (0214-445) is gratefully acknowledged.

E-mail address: markus@princeton.edu 


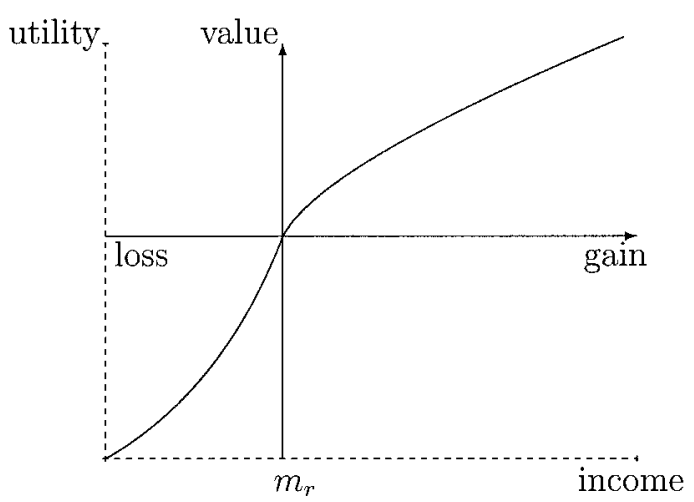

FIGURE 1. Value function of Tversky and Kahneman.

(iii) Individuals exhibit a diminishing sensitivity to losses. An increase in a small monetary loss leads to a far higher decrease in utility than an increase in a monetary loss that was already large. People become more willing to take on risk after suffering a substantial loss. More specifically, they become less risk averse or even risk loving over losses. This translates into a value function which is less concave or even convex in the loss region.

These findings are documented in Kahneman and Tversky's (1979) seminal work on Prospect Theory. Prospect Theory divides an individual's choice process into two phases: a framing/editing phase, in which individuals simplify and narrow their decision problem, and an evaluation phase. For the latter phase, Kahneman and Tversky (1979) develop a value function as depicted in Figure 1 using the certainty equivalence method. Following Markowitz (1952), this value function is defined over gains and losses rather than over absolute income. This value function can also be viewed as a reference-dependent utility function where the reference income level is $m_{r}$

The aim of this paper is to explain observed features of an individual's risk attitude with a minimal departure from the standard von Neumann-Morgenstern utility setup. The standard model implicitly assumes that people can immediately find their new optimal consumption bundle as their income changes. After sudden changes in income, decision makers have to choose a totally new optimal consumption bundle. However, choosing an optimal consumption bundle given a choice of thousands of commodities is not an easy task. Our model describes a boundedly rational decision maker who has to learn and hence is unable to immediately find her optimal consumption bundle with certainty. While the choices of rational agents are only constrained by their lack of information, boundedly rational decision makers are in addition restricted in their ability to process the available information. This can occur because they 
apply simpler but less precise computational methods in order to save information processing costs and time.

The incorporation of these cognitive elements into the standard model alters the decision maker's attitude towards income lotteries. We show that bounded rationality increases risk aversion at the reference income level and there exists a range of income levels below the reference income where bounded rationality reduces risk aversion. Hence, we provide a theoretical explanation for why people become less risk averse after they have faced an unexpected loss. Our model also generates the reference-dependent preference setting that was formally introduced in Tversky and Kahneman (1991). ${ }^{1}$

The structure of this paper is as follows. Section 2 provides the intuition behind our model. The formal analysis is presented in Section 3. Conclusions and potential applications of our model are presented in Section 4.

\section{Intuition}

A boundedly rational decision maker may not be able to consume her optimal consumption bundle after an unexpected income change. There are at least two reasons why this might occur. First, she is unable to choose her optimal bundle (with certainty), and second, she does not know her truly optimal bundle because of limitations in her information processing abilities.

In the first scenario, the decision maker tries to consume her newly optimal consumption bundle after the income change, but ends up not buying the targeted bundle with certainty. This scenario serves as a metaphor for several realistic features of consumer behavior. For example, in reality people buy commodities sequentially rather than buying them all at once. If they temporarily forget that their income has declined, they might unconsciously buy quantities that they are used to. Thus, when the decision maker is faced with a new unfamiliar income level, she might end up buying too many of the goods that she encounters first. In that case, she will not have enough money left for the remaining commodities. Similarly, after winning a lottery, people initially become overconfident and they may overestimate how much they can afford to spend. Another reason why people might depart from their optimal consumption plan is temptation. While we are used to dealing with temptation at our familiar income level, the appropriate commitment devices that ensure that we stick to our original consumption plan might not be in place at a new income level. Durable goods that require monthly installments provide yet another example.

1. It should be pointed out that there are other experimental findings, such as framing effects, which cannot be brought in line with von Neumann-Morgenstern axioms. Alternative theories are needed to address these issues. Camerer (1995) and Hey (1997) provide nice summaries of this literature. 


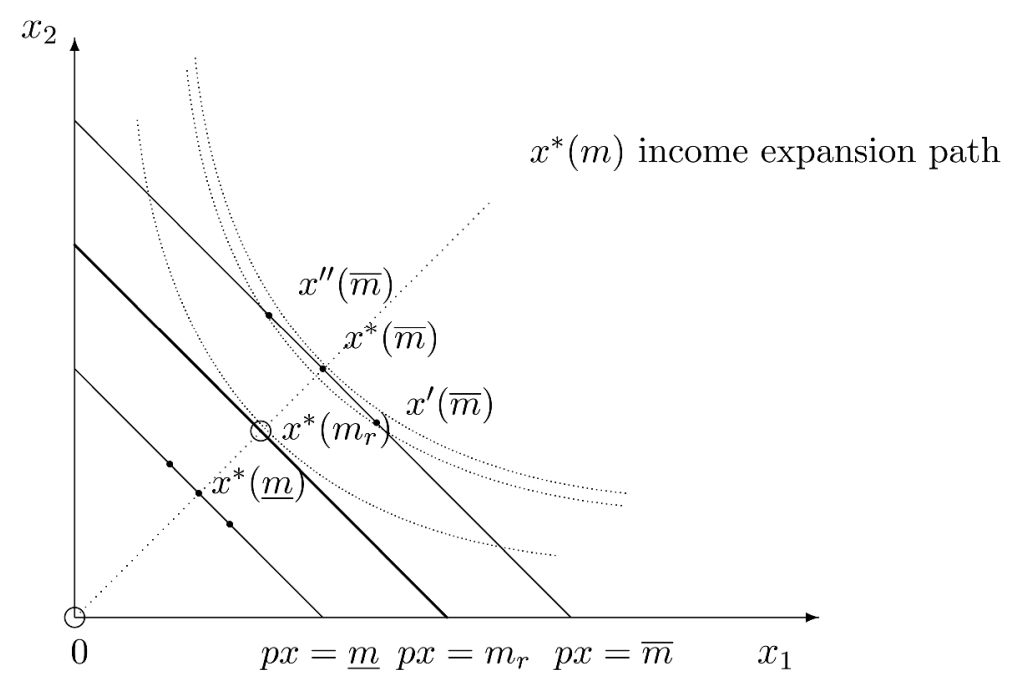

FIGURE 2. Direct utility.

If an agent has purchased durable goods prior to the income change, she might not be able to optimally adjust her consumption pattern after the change. In general, any error in arithmetic or other bias might serve as a source of bounded rationality and lead to a nonoptimal choice of consumption bundle. The random choice approach, presented in Section 3.1, illustrates how these errors affect the decision makers' risk aversion.

In the second scenario, the decision maker is able to pick her targeted bundle without any error, but she does not know her truly optimal consumption bundle for unfamiliar income levels. In other words, she is aware of the fact that there might exist a bundle from which she would derive a higher experienced utility. The random utility approach developed in Section 3.2 captures this feature by introducing random state-dependent utility functions.

Since both approaches share a similar intuition, we illustrate the main arguments that follow in this section within the context of the random choice approach. While the decision maker will not choose her optimal consumption bundle with certainty after an income change, she is very familiar with her optimal bundle at her reference income level $m_{r}$. Given this experience it is reasonable to assume that she will not make errors at her reference income level.

Figure 2 shows an individual's consumption choice problem for three different income levels $\underline{m}, m_{r}, \bar{m}$. A rational decision maker always picks his optimal consumption bundle $x^{*}(m)$ at any income level $m$. That is, his indifference curve is tangent to the budget line for any income level. His corresponding indirect utility function $v(m)$ is depicted in Figure 3. On the other hand, a boundedly rational decision maker is only able to consume the optimal bundle 


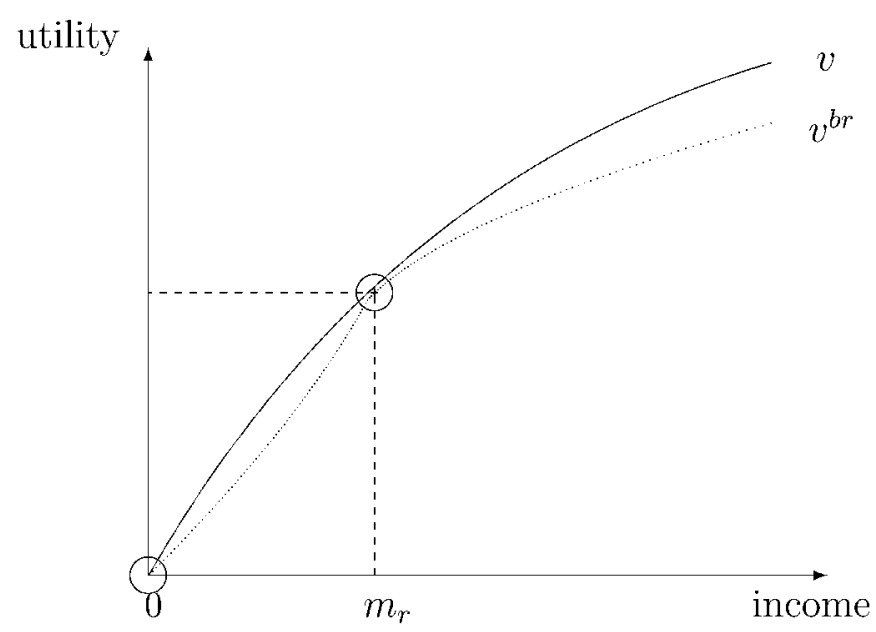

FIGURE 3. Indirect utility of rational and boundedly rational agent.

at her reference income level $m_{r}$ (middle budget line). At this income level, she does not make any errors and, hence, her indirect utility level $v^{\mathrm{br}}\left(m_{r}\right)$ is as high as the one of an identical rational decision maker. For any other positive income level different from $m_{r}$, she is not able to obtain the optimal consumption bundle with probability one. For example, at the higher income level $\bar{m}$, she tries to consume $\hat{x}(\bar{m})=x^{*}(\bar{m})$ but makes a random error in selecting the consumption bundle and, hence, she ends up consuming $x^{\prime}(\bar{m})$ or $x^{\prime \prime}(\bar{m})$. Both bundles lie on an indifference curve that yields lower utility than the one passing through $x^{*}(\bar{m})$. Consequently, her indirect utility function $v^{\mathrm{br}}(\bar{m})$ is strictly lower than the one of the rational decision maker. The same argument applies for a lower income level $\underline{m}$ and any other strictly positive income level different from $m_{r}$. One can also view the resulting utility loss due to choice of a nonoptimal consumption bundle at other income levels as an implicit cost of reoptimization. A more direct approach would be to assume these costs of reoptimization directly.

Figure 3 shows that since the boundedly rational decision maker's indirect utility function is strictly lower than that of an identical rational agent around $m_{r}$ but equal at the reference income level $m_{r}$, her utility $v^{\text {br }}(m)$ must be more concave than $v(m)$ (and may even be kinked) around $m_{r}$. In other words, she is locally more risk averse at her reference income level than an identical rational decision maker. Hence, this setting provides a natural explanation for the dependence of agents' risk attitudes on the reference income level and the high risk aversion at the reference income level. The reason for this loss aversion in our setting is that if a boundedly rational decision maker accepts a lottery, she 
actually faces a compound lottery. At the first stage, a lottery outcome over income is drawn, and at the second stage she faces another lottery caused by the error she makes in choosing a consumption bundle. The latter stage always has a negative expected value because she can only worsen her situation by making errors.

Note from Figure 2 that as the income declines the budget line shortens and with it the potential departure from the optimal bundle is reduced. This purely stems from the fact that one cannot consume a negative amount of any good. At the zero-income level, the decision maker cannot choose a nonoptimal consumption bundle since she simply has no income to spend. ${ }^{2}$ Hence, $m=0$ forms the second focal point-marked by a circle - where the indirect utility functions of the boundedly rational and the rational decision maker coincide. From Figure 3 one can easily see that as $m$ declines starting from $m_{r}, v^{\text {br }}$ declines faster than $v$ to guarantee that $v^{\text {br }}<v$ for $m \in\left(0, m_{r}\right)$. To ensure that both differentiable functions end up at the same utility level at $m=0$ it has to be the case that there exists a range of income levels between 0 and $m_{r}$ where $v^{\text {br }}$ is less concave or even convex. In other words, bounded rationality reduces risk aversion over this income range. This result corresponds with the experimental observations of diminishing sensitivity.

Additional insights can be gained by relating these results to consumer theory. The indirect utility function $v^{\text {br }}(m)$ can be thought of as resulting from maximization behavior subject to an additional constraint given by the error term. Given that the indirect utility function of an identically rational individual $v(m)$ is the envelope of $v^{\mathrm{br}}(m)$ with tangent point at the reference income level, the increased risk aversion at $m_{r}$ follows naturally. This is in the same vein as the Le Châtelier principle, especially if one assumes that the decision maker learns to choose the new optimal consumption bundle over time. The Le Châtelier Principle states that the response of optimized variables to a small structural change to the system is reduced if more constraints are added to how the variables can be changed. In our case $x^{*}(m)$ maximizes the utility function for a given income, $m$. The additional random errors can be thought of as additional constraints on how $x^{*}(m)$ can change as $m$ departs from $m_{r}$.

This discussion also suggests that a decision maker is more risk averse if she has spent a large amount of her money on durable goods. A sudden

2. Literally interpreted, the bundle $x$ consists of all consumption goods and $m$ reflects the total income of the decision maker. A different interpretation is possible in a setting where the decision maker lexicographically prefers a certain level of basic goods, like food and housing, before demanding extra treats, like restaurant visits and luxury goods. As long as the worst outcome of the lottery does not cut into the expenditure on everyday needs, one can restrict the vector $x$ to these nonbasic goods. Since agents know their optimal consumption bundle of everyday goods, $m$ can then be viewed as the income earmarked for nonbasic goods. 
income change constrains her from adjusting to the new optimal consumption bundle. She still has to consume the durable commodities that she bought in the previous periods. It is interesting that the expected riskiness of the income stream together with her risk aversion determines the amount she is willing to spend on durable commodities, which, in turn, influences her risk aversion.

\section{Formal Analysis}

Before we formally develop the random choice and random utility approaches, let us first make some rather standard assumptions on the decision maker's preferences and their utility representation. We assume that the decision maker is still fairly rational since she has a transitive preference ordering that satisfies the von Neumann-Morgenstern axioms.

\section{AsSUMPTION 1.}

(i) The utility function $u: \mathbb{R}_{+}^{k} \rightarrow \mathbb{R}$, represents a complete, reflexive and transitive preference ordering over the commodity space $\mathbb{R}_{+}^{k}$ of $\mathrm{k}$ different goods.

(ii) $u$ is a von Neumann-Morgenstern utility function. That is, the decision maker prefers random commodity bundle $\tilde{x}$ to $\tilde{x}^{\prime}$ if and only if $E[u(\tilde{x})] \geq$ $E\left[u\left(\tilde{x}^{\prime}\right)\right]$.

(iii) $u$ is weakly increasing, and strictly increasing in at least one of its arguments.

(iv) $u$ is twice differentiable, and all resulting indirect utility functions are also well defined and twice differentiable.

The assumption that the utility functions are twice differentiable allows us to use the Arrow-Pratt risk aversion measure.

\subsection{Random Choice Approach}

Let us now depart from the standard utility maximization problem and assume that the decision maker might make errors in her consumption choice since she applies heuristics instead of exact maximization procedures. She tries to consume target bundle $\hat{x}$ but ends up consuming $\mathrm{x}^{\mathrm{br}}$. That is, the actual consumption bundle chosen given an income of $m$ and a reference income level $m_{r}$ is

$$
x^{b r}\left(\hat{x}, m_{r}\right) \equiv \hat{x}\left(m, m_{r}\right)+\underline{e}\left(\hat{x}, m_{r}\right),
$$

where $\hat{x}$ is the target bundle the decision maker tries to achieve and $\underline{\tilde{e}}$ is the error captured by a k-dimensional random function. All terms depend on the reference 
income level $m_{r}$. Since we will not vary $m_{r}$ in our analysis, we will drop it as an argument.

Note that the decision maker need not necessarily aim for an optimal bundle, $x^{*}(m) \in X^{*}(m) \equiv \arg \max \{u(x)$ s.t. $p x \leq m\}$, given that she knows the distribution of the error term. This is especially the case when the error term is biased. If, for example, the decision maker always ends up accidentally buying too much chocolate, it is probably useful for her to aim at a consumption bundle with less chocolate than in the optimal consumption bundle. Therefore, an optimal target bundle to aim for is given by

$$
\hat{x}^{*}(m) \in \arg \max \{E[u(\hat{x}+\underline{e}(\hat{x}))] \text { s.t. } p \hat{x} \leq m\}
$$

Let us simplify the notation to $\tilde{e}(m) \equiv \underline{e}\left(\hat{x}^{*}(m)\right)$, before imposing some restrictions on the error term $\underline{e}(\cdot)$.

Assumption 2. The error term $\tilde{\mathrm{e}}(\cdot)$ is such that

(i) it does not lead to negative consumption. That is, $x^{\text {br }} \subset \mathbb{R}_{+}^{k}$;

(ii) any $x^{\text {br }}$ is affordable and hence within the budget set;

(iii) the decision maker can choose her 'familiar' bundle without error at her reference income level. Formally, there exists $\hat{x}^{*}\left(m_{r}\right) \in X^{*}\left(m_{r}\right)$ s.t. $e\left(m_{r}\right)=$ 0 ;

(iv) an error occurs with positive probability, that is, $\operatorname{Pr}\left[x^{\mathrm{br}}(\hat{x}) \in X^{*}(m)\right]<1$ unless it is ruled out by (i)-(iii);

(v) all realizations of $e(\cdot)$ are twice differentiable in $\mathrm{m}$ to guarantee smoothness; and

(vi) the decision maker can always choose to spend less money on everything else and spend the rest on the good i from which she derives strictly positive marginal utility.

Assumption 2(i) rules out negative consumption for any commodity. Assumption 2(ii) guarantees that the errors are such that the decision maker does not spend more than her income. Assumption 2(iii) assumes that the decision maker knows how to choose the optimal consumption bundle at the reference income level. In other words, she makes no errors at the reference income level, $m_{r}$. Assumption 2(iv) states that the boundedly rational decision maker usually has trouble finding her optimal consumption bundle. Assumption 2(vi) rules out the possibility that an increase in income makes the decision maker worse off. In other words, the increase in the error due to higher income has a lower impact on the expected utility than the enlargement of the budget set. This assumption is plausible since the decision maker does not need to spend all of her income. Consequently, higher income does no harm. In addition, we assume that the decision maker is sufficiently rational that she can spend the remaining income without error 
on a special commodity $i$ that leads to a strict increase in her utility. Consequently, she will always strictly prefer higher income and spend all her money.

To clarify the analysis, let us define the indirect utility function $v(m) \equiv$ $u\left(x^{*}(m)\right)$ of an equivalent rational decision maker, who always chooses the optimal consumption bundle $x^{*}(m) \in \arg \max \{u(x)$ s.t. $p x \leq m\}$. Thus, $v(m)$ contrasts with the indirect utility function, $v^{\mathrm{br}}(m) \equiv E\left[u\left(\hat{x}^{*}(m)+\tilde{e}(m)\right)\right]$, of a boundedly rational decision maker. The functional $f(\cdot)$ links both indirect utility functions by mapping $v(m)$ onto $v^{\text {br }}(m)$.

Definition 1 defines the standard Arrow-Pratt measures of (absolute) risk aversion for the rational decision maker whose indirect utility function is $v(m)$ and for the boundedly rational decision maker whose indirect utility function is $v^{\mathrm{br}}(m)$. The difference between both measures $c(m)$ isolates the part of risk aversion which is due to bounded rationality.

\section{DEFINITION 1.}

(i) The Arrow-Pratt measures of (absolute) risk aversion for the indirect utility functions is

$$
r(m) \equiv-\frac{\partial^{2} v / \partial m^{2}}{\partial v / \partial m}
$$

for the rational decision maker and

$$
r^{\mathrm{br}}(m) \equiv-\frac{\partial^{2} v^{\mathrm{br}} / \partial m^{2}}{\partial v^{\mathrm{br}} / \partial m}
$$

for the boundedly rational decision makers.

(ii) The risk aversion contribution of bounded rationality, c, is

$$
c(m) \equiv r^{\mathrm{br}}(m)-r(m) .
$$

The definition of $c(m)$ allows us to separate risk aversion into two parts, one being the actual risk aversion given by the concavity of the utility function and the other being the risk aversion contribution of bounded rationality (induced by the optimal consumption bundle not being chosen). We will express the risk aversion contribution in terms of the functional $f(v)$ to simplify the proofs that follow. In the Appendix we derive the equation

$$
c(m)=-\frac{\partial^{2} f / \partial v^{2}}{\partial f / \partial v} \frac{\partial v}{\partial m} .
$$

A similar calculation can be found in Pratt (1964).

Lemma 1 shows that an error strictly reduces the decision maker's expected utility since a nonoptimal commodity bundle is consumed with 
positive probability. This is not the case at two income levels that we label focal points. At a zero income level no consumption takes place and hence the decision maker cannot err. At the reference income level $m_{r}$ the decision maker does not make mistakes since he has learned how to choose the optimal consumption bundle at that level. All proofs are presented in the Appendix.

LEMMA 1. A focal point is an isolated income level where bounded rationality has no impact on the utility level. At all other income levels, bounded rationality strictly reduces the indirect utility function. The focal points are 0 and $m_{r}$. That is,

(i) $v^{\mathrm{br}}(m)=v(m)$ for $m \in\left\{0, m_{r}\right\}$

(ii) $v^{\text {br }}(m)<v(m)$ for $m \in \mathbb{R}_{+} \backslash\left\{0, m_{r}\right\}$.

Lemma 1 illustrates that the indirect utility function of an identical rational decision maker (who makes no errors) serves as an upper envelope for the indirect utility function of the boundedly rational agent. The two focal points 0 and $m_{r}$ are illustrated in Figure 3. The focal point 0 depends on the Assumption 2(i) $x^{\text {br }} \subset \mathbb{R}_{+}^{k}$, which states that consumption of any commodity cannot be negative. This binds the space for the error term. If income decreases, this space also decreases. At zero income level, the possible consumption set is the single point 0 , i.e., the error term vanishes. In other words, there is no possibility to err at a zero income level since only zero consumption (of non-basic goods) is possible at that level. As pointed out in footnote 2, for small lotteries the income $m$ can also be viewed as the income intended for non-basic goods, like extra treats, if the decision maker lexicographically prefers a certain level of everyday goods over them.

The shape of $v^{\text {br }}$ is also plausible in a truly dynamic learning model. Before the decision maker has learnt how to choose her optimal consumption bundle at the reference income level, $x^{*}\left(m_{r}\right)$, her expected utility is strictly below $v(m)$ for all positive income levels. After she knows $x^{*}\left(m_{r}\right)$, her utility level for incomes around $m_{r}$ increases, leading to the indirect utility function $v^{\mathrm{br}}(m)$ illustrated in Figure 3.

Using Lemma 1, we can show that bounded rationality, defined in this subsection as making small errors in choosing the optimal commodity bundle, increases risk aversion at the reference income level.

PROPOSITION 1. Bounded rationality increases absolute risk aversion at the reference income level. More specifically, 
(i) the risk aversion contribution coefficient is positive, i.e., $c\left(m_{r}\right) \geq 0$;

(ii) a boundedly rational decision maker strictly prefers the reference income level, $m_{r}$, to any nondegenerated lottery whose certainty equivalence for an identical rational decision maker is $m_{r}$

The high risk aversion at the reference income level $m_{r}$ is in line with the experimental findings of loss aversion. For any outcome of the lottery which is different from 0 or $m_{r}$, the decision maker has to incur costs to find the new optimal consumption bundle, which she does not achieve with certainty. Therefore, lotteries are less attractive for a boundedly rational decision maker. ${ }^{3}$ Proposition 1 also highlights the importance of the reference point for analyzing risk behavior. The reference point also appears in related findings such as the status quo bias and the endowment effect. ${ }^{4}$

Relaxing the assumption that the error term $e(m)$ is twice differentiable could induce a kink in the indirect utility function $v^{\text {br }}$ at $m_{r}$. For example if $e(m)$ were V-shaped at $m_{r}$, a kink could arise at $v^{\mathrm{br}}\left(m_{r}\right)$. However, a nondifferentiable error function $e(m)$ has the disadvantage that the Arrow-Pratt risk aversion measure is not well defined. In that case, local risk aversion can be measured by using the preference ordering over $\epsilon$-income lotteries by comparing their certainty equivalence. It is easy to see that Proposition 1 still holds for this case. ${ }^{5}$

While Proposition 1 shows that bounded rationality increases risk aversion at the reference income level, Proposition 2 claims that bounded rationality decreases risk aversion or even leads to risk loving behavior at a lower income level. The risk-seeking attitude in parts of the loss region can be attributed to diminishing sensitivity.

PROPOSITION 2. There exists a range of incomes $(\underline{m}, \bar{m})$ between the two focal points 0 and $m_{r}$ where bounded rationality reduces risk aversion or leads to risk loving behavior. That is, $c(m)<0$ for some income range in $(\underline{m}, \bar{m})$.

As explained earlier, this result is driven by the fact that only smaller errors are possible at a lower income level. A lower income reduces the budget set within which the consumption bundle that is eventually chosen, $x^{\mathrm{br}}=\hat{x}^{*}+\tilde{e}$, has to lie.

The range of income levels $(\underline{m}, \bar{m})$ where risk aversion decreases because of bounded rationality is determined by both the error term and the utility function.

3. As pointed out in Section 2, the costs of reoptimization after an income change might be due to durable goods. Koo and Singh (1998) independently derive a similar result to Proposition 1 for the nontradable good case.

4. See Rabin (1998) for an explanation of the differences between loss aversion, status quo bias and endowment effect.

5. However, it would considerably increase the notational burden. 
There are three factors determining the size and location of that range of income levels. First, since indifference curves at lower income levels are generally more curved, the same error causes a higher disutility at a lower income level. Second, the error possibility space shrinks as income decreases. In the extreme case of a zero income, there is no space left for any error. One can show that the degree to which the error possibility space shrinks depends on the number of available commodities. Third, the distance to the reference income level increases as income decreases. It is plausible that the variance of the error term increases with this distance. A larger variance in turn leads to a lower utility level for strictly quasi-concave utility functions. All three factors influence the size and location of this income range. Whereas the first factor suggests that the relevant range should be further away from the reference income, the second suggests the opposite. The third factor pushes $(\underline{m}, \bar{m})$ closer to $m_{r}$ if the variance of the error term increases concavely as the distance from the reference income level increases.

Note that there might be more than one income region where bounded rationality reduces risk aversion. This is particularly the case when the variance of the error term $e(m)$ does not have an inverse U-shape between $m=0$ and $m_{r}$.

\subsection{Random Utility Approach}

The random choice approach presented in Subsection 3.1 assumes that the decision maker knows her optimal consumption bundle at each income level but is unable to pick it with certainty. However, in reality a decision maker does not know her truly optimal bundle for each possible income level. She will only find the bundle that yields the highest experienced utility at this income level after experimenting with different consumption bundles at a given income level. To capture this fact, we introduce state-dependent utility functions $u_{s}(x) .{ }^{6}$ Which bundle leads to the highest experienced utility for a given income $m$ depends on the ex-post realization of the state $s$. For simplicity, we assume that $s$ is independently distributed of $m$. In other words, after the decision maker has won or lost a lottery, she has to choose her new consumption bundle for her new income level without knowing the realization of the state $s$ upon which her utility function depends. She might only realize that she has not chosen the optimal bundle for her new income level after she has consumed the new bundle. At her reference income level $m_{r}$ we assume that all possible $u_{s}(x)$ lead to the same optimal consumption. She can rule out other utility functions $u_{s}(x)$ without this property since she knows which bundle leads to the highest experienced utility at $m_{r}$. This assumption-as formalized in Appendix A.3

6. In Chen, Friedman, and Thisse (1997) a related random utility approach is applied to game theoretic settings. 
(ii-a) - is justified by the fact that the decision maker has faced the same choice problem many times before and hence has figured out the optimal bundle at $m_{r}$ At all positive income levels different from $m_{r}$ Assumption 3(ii-b) rules out the case where all possible utility functions $u_{s}(x)$ would lead to the same optimal consumption bundle.

\section{AsSUMPTION 3.}

(i) Each state-dependent utility function $u_{s}(x)$ satisfies Assumptions 1 and each state $s$ occurs with strictly positive probability.

(ii) All state-dependent optimal bundles $x_{s}^{*}(m) \in \arg \max \left\{u_{s}(x)\right.$ s.t. $\left.p x \leq m\right\} \equiv$ $X_{s}^{*}(m)$

(a) coincide at the reference income level $m_{r}$, that is $x_{s}^{*}(m)=x_{m_{r}}^{*}$ for all states $s$;

(b) do not coincide for all income levels $m \in \mathbb{R}_{+} \backslash\left\{0, m_{r}\right\}$. That is, $\bigcap_{s} X_{s}^{*}(m)=\varnothing$.

Note that even though the decision maker does not know her state-realized utility function $u_{s}(x)$, she still has a complete preference ordering over the whole commodity space represented by $E_{s} u_{s}(x)$.

The effects on risk aversion in both approaches are driven by the fact that the decision maker does not choose the optimal consumption bundle with probability 1 . This similarity already indicates that the results presented for the random choice approach also hold for the random utility approach. However, there are differences between the two approaches. In the random choice approach, the commodity bundle that is actually consumed, $x^{\text {br }}=\hat{x}$ $+e$, is random. In the random utility approach, the consumed bundle $x^{\mathrm{BR}}(m)$ $\equiv \arg \max \left\{E_{s} u_{s}(x)\right.$ s.t. $\left.p x \leq m\right\}$ is nonstochastic, while the optimal bundle $x_{s}^{*}(m)$ is state-dependent and hence random. ${ }^{7}$ Notice that the difference $x^{\mathrm{BR}}(m)-x_{s}^{*}(m)$ serves the same role as the error term $e(m)$ in the random choice approach.

Furthermore, in the random utility setting we have to specify whether the identical rational decision maker, who serves as a benchmark, knows his $u_{s}$ already (i) prior to his decision to accept/reject the lottery, or only (ii) prior to his consumption choice. In the former case, the benchmark rational indirect utility function is only unique if we assume in addition that all statedependent utility functions $u_{s}(x)$ will lead to the same (rational) indirect utility function, i.e., $u_{s}\left(x_{s}^{*}(m)\right)=v(m)$ for all $s$. To avoid this assumption, we choose the latter case as the benchmark. That is, the rational decision maker does not know his utility function at the time of his lottery choice, but

7. The superscript "BR" in capital letters refers to the bounded rational decision maker in the random utility approach. Similarly, we replace $r, r^{\mathrm{br}}$, and $c$ with their equivalents in capital letters to distinguish the random utility setting from the random choice setting. 
he will know it before he makes his final consumption decision. More formally, his expected indirect utility function is given by $E[v(m)]=$ $E_{s}\left[u_{s}\left(x_{s}^{*}(m)\right)\right]$. Having defined the benchmark, the analysis can be conducted analogously to the random choice approach. It is easy to see that the indirect utility function of a boundedly rational decision maker $E\left[v^{\mathrm{BR}}(m)\right] \equiv E_{s}\left[u_{s}\left(x^{\mathrm{BR}}(m)\right)\right]$ is always strictly below $E[v(m)]$ except at the focal points 0 and $m_{r}$. Consequently, the proofs of Propositions 1 and 2 can also be applied to the random utility approach. The proof of Proposition 3 in the Appendix provides the formal argument.

Proposition 3. Propositions 1 and 2 also hold for the random utility approach. In particular, $C\left(m_{r}\right)=R^{\mathrm{BR}}\left(m_{r}\right)-R\left(m_{r}\right)>0$ and there exists a region of income level $(\underline{m}, \bar{m})$ between 0 and $m_{r}$, where $C(m)<0$.

In reality, a boundedly rational decision maker probably does not know her optimal consumption bundle and she errs in choosing it. We do not need any further analysis to see that our results still hold if one combines the random utility approach and the random choice approach.

\section{Possible Extensions and Conclusion}

Our analysis shows that one factor contributing to risk aversion is the fact that the decision maker must find her new consumption bundle after the outcome of the lottery has been realized. This requires that she incurs thinking costs in the realized state of the world. After extending the analysis, it is not surprising that one observes more misjudgement in decisions made about the acceptance of a lottery than in consumption choice. Evaluating a lottery is a much more difficult task because one not only incurs thinking costs in the realized state, but in all possible states. Therefore, the boundedly rational decision maker will apply a simpler heuristic in evaluating a lottery.

A related area of research examines the question of finding the optimal planning horizon in a world with uncertainty. Planning for a distant future increases the number of states exponentially, which makes the maximization problem much more complicated. Therefore, boundedly rational decision makers will apply a heuristic that is less precise for long-horizon problems than for short-term problems. It remains to be shown that the optimal heuristic provides a fairly exact prediction for the near future and a rougher prediction for the distant future. It also seems plausible that increasing uncertainty levels make people more shortsighted, which can explain why high volatility in the inflation rate, i.e., price uncertainty, hurts the economy. The optimal planning horizon solution also provides an explanation for the 
demand for flexibility or liquidity and for why we observe incomplete contracts.

The model can be extended to include uncertainty in both income and prices. It is a well-known fact that in traditional microeconomic models where the decision maker's utility function is quasi-concave and exhibits constant marginal utility of income, the decision maker is risk loving with respect to price uncertainty. This is due to the fact that she chooses her optimal consumption bundle after the prices are realized. In an analysis with error possibilities similar to ours, this risk-loving behavior need not be true.

\section{Appendix}

A.1. Proof of $c(m)=-\frac{\partial^{2} f / \partial v^{2}}{\partial f / \partial v} \frac{\partial v}{\partial m}$

Note that $v(m)$ is strictly increasing in $m$ due to Assumption 1(iii). Consequently, the inverse of $v(m)$ exists and is denoted by $h(v(m))=m$. The functional $f(\cdot)$ is given by $f(v)=v^{\mathrm{br}}(h(v))$. Since $v(m)$ is twice continuously differentiable in $m$, so is $h(v)$ and $f(v)$.

$$
\begin{aligned}
\frac{\partial v^{\mathrm{br}}}{\partial m} & =\frac{\partial f}{\partial v} \frac{\partial v}{\partial m}, \\
\frac{\partial^{2} v^{\mathrm{br}}}{\partial m^{2}} & =\left(\frac{\partial^{2} f}{\partial v^{2}} \frac{\partial v}{\partial m}\right) \frac{\partial v}{\partial m}+\frac{\partial f}{\partial v} \frac{\partial^{2} v}{\partial m^{2}}-\frac{\partial^{2} v^{\mathrm{br}} / \partial m^{2}}{\partial v^{\mathrm{br}} / \partial m} \\
& =-\frac{\partial^{2} f / \partial v^{2}}{\partial f / \partial v} \frac{\partial v}{\partial m}-\frac{\partial^{2} v / \partial m^{2}}{\partial v / \partial m} \\
r^{\mathrm{br}}=c+r . &
\end{aligned}
$$

\section{A.2. Proof of Lemma 1}

(i) (1) For $m=0$

Since $x_{s}^{\mathrm{br}} \in \mathbb{R}_{+}^{k}$ and $p x_{s}^{\mathrm{br}}=m=0 \forall s \forall x_{s}^{\mathrm{br}}, x_{s}^{\mathrm{br}}(0)=x^{*}(0) \forall s$.

(2) For $m=m_{r}$

By Assumption 2(iii), $\underline{e}_{s}\left(x_{i}^{*}\left(m_{r}\right)\right)=0 \forall s$. Hence, $x^{\mathrm{br}}\left(\hat{x}_{i}^{*}\left(m_{r}\right)\right) \in X^{*}\left(m_{r}\right)$.

(ii) For each $m \in \mathbb{R}_{+} \backslash\left\{0, m_{r}\right\}$

By Assumption 2(iv), $\operatorname{Pr}\left(x^{\mathrm{br}}\left(\hat{x}(m) \in X^{*}(m)\right)<1\right.$ and thus $v(m)>v^{\mathrm{br}}(m)$. 


\section{A.3. Proof of Proposition 1}

(i) Since

$$
c(m)=-\frac{\partial^{2} f / \partial v^{2}}{\partial f / \partial v} \frac{\partial v}{\partial m}
$$

it is sufficient to determine the sign of the three factors.

(1) $\partial v / \partial m>0$, since $u(x)$ is strictly increasing in at least one argument. Let $g(v(m)) \equiv f(v(m))-v(m)$. Since $f(\cdot)$ and $v(m) \in C^{2}, g(\cdot) \in C^{2}$. By Lemma $1, g(\cdot)$ has a local maximum at $v\left(m_{r}\right)$. Therefore, $\partial g /\left.\partial v\right|_{v\left(m_{r}\right)}=0$ and $\partial^{2} g /\left.\partial v^{2}\right|_{v\left(m_{r}\right)} \leq 0$, which yields

(2) $\left.\frac{\partial f}{\partial v}\right|_{v\left(m_{r}\right)}=1>0$,

(3) $\left.\frac{\partial^{2} f}{\partial v^{2}}\right|_{v\left(m_{r}\right)} \leq 0$.

(ii) Take any lottery $\tilde{m}$ (with distribution $F$ ) whose certainty equivalence for a rational decision maker is $m_{r}$, i.e., $E_{F}[v(\tilde{m})]=v\left(m_{r}\right)$. By Lemma 1 $v^{\mathrm{br}}\left(m_{j}\right)<v\left(m_{j}\right)$ for any realization $m_{j}$ of $\tilde{m} \notin\left\{0, m_{r}\right\}$. Thus, $E_{F}\left[v^{\mathrm{br}}(\tilde{m})\right]<$ $E_{F}[v(\tilde{m})]=v\left(m_{r}\right)=v^{\text {br }}\left(m_{r}\right)$.

\section{A.4. Proof of Proposition 2}

$c(m)=-\frac{\partial^{2} f / \partial v^{2}}{\partial f / \partial v} \frac{\partial v}{\partial m}$. It is sufficient to determine the sign of the factors.

(1) $\partial v / \partial m>0$ as shown in the proof of Proposition 1.

(2) $\partial f / \partial v>0$, since $\partial v^{\mathrm{br}} / \partial m=(\partial f / \partial v)(\partial v / \partial m)$ and $v$ and $v^{\mathrm{br}}$ are strictly increasing in $m$.

(3) $\exists(\underline{m}, \bar{m}) \subset\left(0, m_{r}\right)$, s.t. $\partial^{2} f / \partial v^{2}>0$ on $(v(\underline{m}), v(\bar{m}))$.

This is shown in the following three steps:

(3.1) $\exists a \subset\left(v(0), v\left(m_{r}\right)\right)$ s.t. $\partial^{2} f /\left.\partial v^{2}\right|_{\mathbf{a}}>0$.

By Lemma $1, f(v(0))=v(0)$ and $f(v(\varepsilon))<v(\varepsilon)$ for sufficiently small $\varepsilon>0$. From this we can conclude that $\partial f /\left.\partial v\right|_{v(\varepsilon / 2)}<1$. We also know from Proposition 1 that $\partial f /\left.\partial v\right|_{v\left(m_{r}\right)}=1$. Applying the mean value theorem on $\partial f / \partial v(\cdot), \exists a \in\left(v(\varepsilon / 2), v\left(m_{r}\right)\right)$ such that

$$
\left.\frac{\partial^{2} f}{\partial v^{2}}\right|_{a}=\frac{\overbrace{\partial f /\left.\partial v\right|_{v\left(m_{r}\right)}}^{=1}-\overbrace{\partial f /\left.\partial v\right|_{v(\varepsilon / 2)}}^{<1}}{\underbrace{v\left(m_{r}\right)-v(\varepsilon / 2)}_{>0}}>0 .
$$


(3.2) Since $f$ is twice differentiable and $\partial^{2} f / \partial v^{2}>0$ at $a$, the latter must be also true at $\left(a-\varepsilon^{\prime}, a+\varepsilon^{\prime}\right)$ for small $\varepsilon^{\prime}>0$.

(3.3) Since $v(\cdot)$ is strictly increasing and continuous in $m$ there exists for each $\vartheta \in\left(a-\varepsilon^{\prime}, a+\varepsilon^{\prime}\right)$ a corresponding $m$ such that $\vartheta=v(m)$.

\section{A.5. Proof of Proposition 3}

$E[v(m)]=E\left[v^{\mathrm{BR}}(m)\right]$ for $m \in\left\{0, m_{r}\right\}$ since $x_{s}^{\mathrm{BR}}\left(m_{r}\right)=x_{s}^{*}\left(m_{r}\right) \forall s$ and $x_{s}^{\mathrm{BR}}(0)=$ $x_{s}^{*}(0)=0 \forall s$.

$E[v(m)]<E\left[v^{\mathrm{BR}}(m)\right]$ for $m \in \mathbb{R}_{+} \backslash\left\{0, m_{r}\right\}$ since $\cap_{s} X_{s}^{*}(m)=\varnothing$ and hence a non-random bundle $x^{\mathrm{BR}}$ cannot be optimal in all states of the world.

$E[v(m)]$ and $E\left[v^{\mathrm{BR}}(m)\right]$ are strictly increasing and twice differentiable since all $u_{s}$ are strictly increasing in at least one argument $x_{i}$ and all $u_{s}$ are twice differentiable.

Hence analogous to the random choice approach, there exists a function $f^{\prime}$ and $C=-\frac{\partial^{2} f^{\prime} / \partial v^{2}}{\partial f^{\prime} / \partial v} \frac{\partial v}{\partial m}$. Since the random utility approach is isomorphic with the random choice approach from this point onwards, it follows immediately that the same results arise.

\section{References}

Camerer, Colin (1995). "Individual Decision Making." In The Handbook of Experimental Economics, edited by John H. Kagel and Alvin E. Roth. Princeton University Press.

Chen, Hsiao-Chi, James W. Friedman, and Jacques-Francois Thisse (1997). "Boundedly Rational Nash Equilibrium: A Probabilistic Choice Approach." Games and Economic Behavior, 18, 32-54.

Hey, John D. (1997). "Experiments and the Economics of Individual Decision Making Under Risk and Uncertainty." In Advances in Economics and Econometrics: Theory and Applications, edited by David M. Kreps and Kenneth F. Wallis. Cambridge University Press.

Kahneman, Daniel and Amos Tversky (1979). "Prospect Theory: An Analysis of Decision Under Risk.” Econometrica, 47, 263-291.

Koo, Hyeng Keun and Rajdeep Singh (1998). "Behavior toward Risk with Non-tradable Commodities," Working paper. Pohang, Korea: Pohang University of Science and Technology and St Louis, Missouri: Washington University.

Markowitz, Harry (1952). "The Utility of Wealth.” Journal of Political Economy, 60, $151-158$.

Pratt, John (1964). "Risk Aversion in the Small and in the Large." Econometrica, 32, 122-136.

Rabin, Matthew (1998). "Psychology and Economics.” Journal of Economic Literature, 36, $11-46$.

Tversky, Amos and Daniel Kahneman (1991). "Loss Aversion in Riskless Choice: A Reference-Dependent Model.” The Quarterly Journal of Economics, 106, 1039-1061. 